\title{
Analysis and Reduction of Electromagnetic Field Leakage through Loaded Apertures: A Numerical Study
}

\author{
OMAR M. RAMAHI
}

Electrical and Computer Engineering Department

University of Waterloo

Waterloo, Ontario, Canada

\section{LIN LI}

Mechanical Engineering Department

CALCE Electronic Products and Systems Center

A. James Clark School of Engineering

University of Maryland

College Park, Maryland, USA

\begin{abstract}
This paper presents the novel use of resistive sheets to reduce electromagnetic penetration through apertures. The resistive material is applied in several configurations that will be discussed in this paper. Significant reduction of electromagnetic leakage is possible by using loading techniques inspired by a transmission line interpretation of the field distribution within the aperture at or beyond its fundamental resonant frequency. By using the configurations proposed in this work, it is possible to achieve appreciable reduction in the near and far field aperture radiation without affecting the total area needed for airflow and heat transfer. Numerical results are presented using a commercial finite-difference time-domain (FDTD) algorithm to demonstrate the effectiveness of our new approach.
\end{abstract}

Keywords electromagnetic compatibility, electromagnetic interference, aperture, coupling, finite-difference time-domain method

\section{Introduction}

The proliferation of high-speed electronic device and communication equipment is resulting in an increasingly hostile electromagnetic environment. Furthermore, the increase in electronic devices sensitivity due to diminishing tolerance levels is increasing the

Received 12 July 2004; accepted 22 February 2005.

Address correspondence to Electrical and Computer Engineering Department, University of Waterloo, Waterloo, Ontario N2T 2H4, Canada. E-mail: oramahi@ece.uwaterloo.ca 
vulnerability of these devices to external electromagnetic threats. In fact, several governmental and nongovernmental research initiatives have been initiated recently to address these critical and important threats to the overall robust operation of electronic equipment in military and civilian settings (see, for example, U.S. Dept. of Defense). The work presented in this paper addresses a critical electronic enclosure design parameter that predominates the effectiveness of electronic enclosures to function as shields of electromagnetic energy.

Metallic enclosures are commonly used to reduce emission from electronic devices and boards and to improve the immunity of the same electronic equipment to external interference and threats. In cases where the frequency is high enough such that the skin depth is less than the thickness of the shield, the overall shielding effectiveness will not be determined by the shield material properties but by the apertures in the shield or chassis. These apertures may be introduced intentionally, as would be the case when airflow is needed for cooling, or unintentionally, as the inevitable joint seams of shields or small openings in doors with imperfect gaskets.

To meet electromagnetic compatibility (EMC) and electromagnetic interference (EMI) requirements, it is crucial to quantify the electromagnetic field penetration through apertures and other chassis openings such as seams and gaskets. The leakage of electromagnetic waves through apertures in enclosures is critical at frequencies where the wavelength approaches the dimension of the aperture.

The problem of radiation through apertures is one of the most classic in the physical sciences. In fact, the fascination with aperture radiation dates back to at least the European Renaissance (Fresnel, 1819/1900; Crew, 1900; Huygens, 1690/1900). Numerous papers have been published in the past fifty years that investigated the behavior of electromagnetic energy as it escapes through apertures. Some of these publications attempted to develop approximate analytical formulations, such as, for instance, the work of Bethe (1944), Hill et al. (1994), and Ott (1998), while other works were not constrained by the electrical size of the aperture and were primarily focused on developing analytical techniques that can be used for a class of aperture configurations (Harrington \& Mautz, 1976; Butler, Rahmat-Samii, \& Mittra, 1978; Gardner \& Costache, 1995; Robinson et al., 1998; Belokour, LoVetri, \& Kashyap, 2001; Cerri, De Leo, \& Primiani, 1992), or efficient numerical techniques that can be used in general (Georgakopoulos, Birtcher, \& Balanis, 2001; Olyslager et al., 1999; Li, Nuebel, Drewniak, DuBroff et al., 2000; Li, Nuebel, Drewniak, Hubing et al., 2000a; Tanabe et al., 1996; Li, Nuebel, Drewniak, Hubing et al., 2000b; Archambeault, 1997). It is perhaps fair to state that most of the works that were published on the radiation of the aperture had to deal with either developing approximate formulae or numerical techniques to predict radiation through apertures. In this work, our objective is to gain additional insight into the underlying aperture radiation mechanism and develop techniques to reduce the radiation without affecting the overall see-through size of the aperture.

In this work, we focus on the problem of radiation through an aperture present in an infinite large conducting plane, thus ignoring any effects induced by the enclosure such as the resonance of the enclosure and complex field interaction with internal structures. This initial focus is intended to develop an understanding for the electric current density distribution in the immediate vicinity of the aperture. To improve shielding effectiveness due to the presence of an aperture in an infinite metallic screen, we introduce a novel technique that is based on the application of resistive sheets (coating) to the area immediately surrounding the aperture. The primary objective in our technique is to lower the radiation through the aperture while maintaining constant aperture size in order not to 


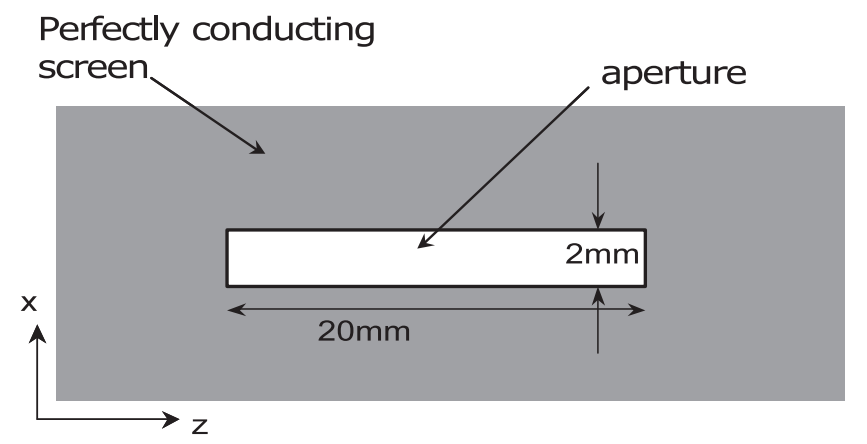

Figure 1. An aperture measuring $2 \mathrm{~mm} \times 20 \mathrm{~mm}$ placed in an infinite perfectly conducting screen.

affect airflow and heat transfer, which is a major consideration for enclosures containing high-speed electronics. The proposed technique is tested using a commercial numerical analysis tool based on the finite-difference time-domain (FDTD) method.

\section{The Aperture as a Transmission Line}

The field mitigation method presented in this work is based on a novel interpretation of the aperture as a transmission line where the power or energy transmission takes place in the plane of the aperture. This interpretation is drastically different from the works in Harrington and Mautz (1976), Butler, Rahmat-Samii, and Mittra (1978), and Gardner and Costache (1995), where the aperture is considered as a load-discontinuity in a transmission line system composed of the interior cavity and exterior open space. However, the interpretation presented here shares similarity with the modeling of the aperture as a short-circuited co-planar transmission line in Robinson et al. (1998). ${ }^{1}$

To motivate this interpretation, we consider an electromagnetic transient wave impinging upon an aperture from one side. The initial part of the transient pulse contains the high-frequency spectrum and is expected to appreciably leak through the aperture. After the initial part of the transient passes through the aperture, one would expect a certain point in time after which some energy starts traveling within the aperture along the directions of the dominant (longer) axis. Naturally, the currents traveling along the edges of the aperture support the field within the aperture. When the field or current reaches the edges of the aperture, it is reflected back. The oscillation of the field (or current) back and forth along the edges terminating the longer axis of the aperture in essence gives rise to what is typically referred to as aperture resonance.

To illustrate this phenomenon further, let us consider an aperture measuring $2 \mathrm{~mm} \times$ $20 \mathrm{~mm}$ placed in an infinite perfectly conducting screen as shown in Figure 1. The aperture resonance phenomenon is observed in Figure 2, which shows three snapshots derived from time-domain simulation of a field incident on the same size aperture. These snapshots show a standing-wave pattern of the field within the aperture at different time instances, a behavior that strongly resembles transmission-line-type propagation. Now, with this physical perspective, we interpret the long edges of the aperture as forming a transmission line system (along which the propagation takes place) and the short edges as

\footnotetext{
${ }^{1}$ The authors acknowledge one of the reviewers who highlighted the co-planar line analogy of the aperture in Robinson et al. (1998).
} 

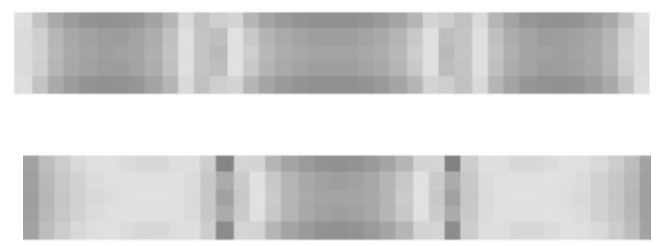

$\mathrm{X}$

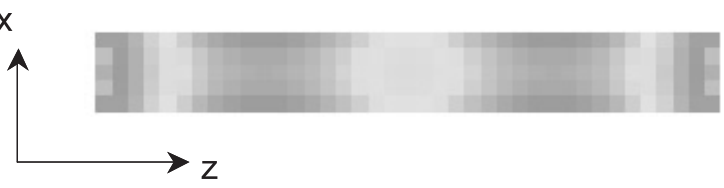

Figure 2. Three different snapshots of the field within the aperture. The excitation is a Gaussian pulse impinging on the aperture from one side.

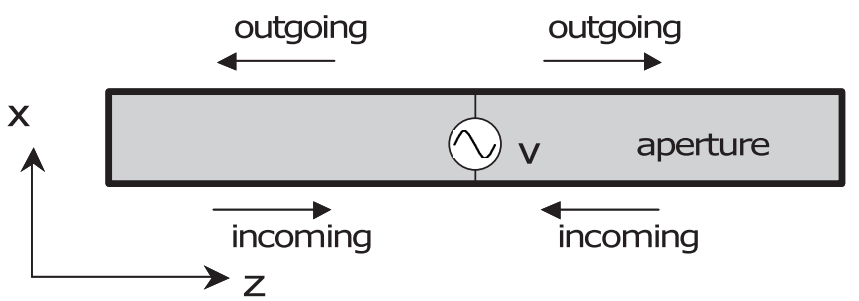

Figure 3. Depiction of the transmission line model of the aperture.

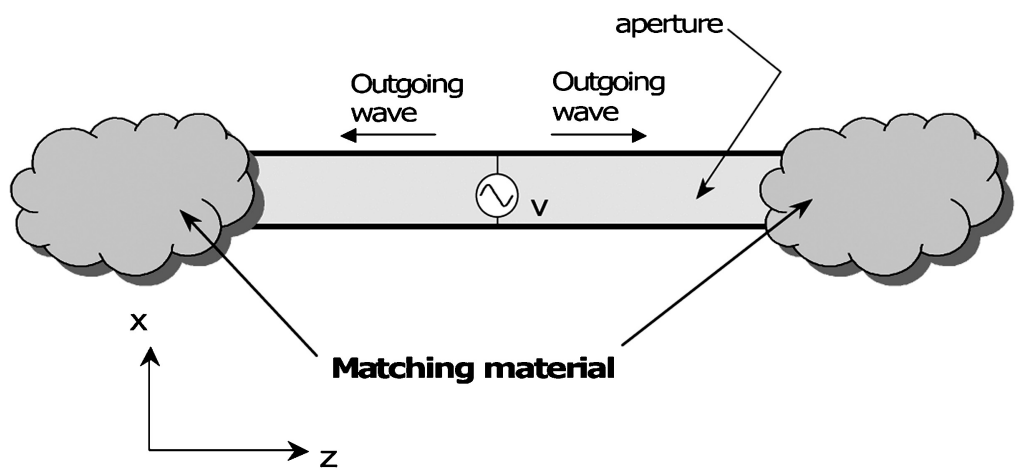

Figure 4. Matching material placed at the short edges of the aperture with the intention of absorbing the outgoing waves.

the termination of the transmission line, as shown in Figure 3. One can assume without any loss of generalization that at resonance, the field excitation is positioned at the middle of the aperture. The field excitation is depicted as a voltage source positioned between the two long edges of the aperture, as shown in Figure 3. As a consequence of this aperture model, if a suitable matching load were positioned at the two ends of the aperture, one would expect that the outgoing fields, and supporting currents on the conducting surface surrounding the aperture, would be absorbed or reduced considerably. In a conceptual perspective, a matching load in this case would be distributed as depicted in Figure 4. 
If a suitable termination were used under this interpretation, the source that is positioned at the center of the aperture would be expected to give rise to outgoing waves (outgoing with respect to the source and in the plane of the aperture).

In light of the above interpretation, the techniques proposed in this work are based wholly on the concept of adding a resistive sheet to the immediate surrounding of the aperture in order to provide a matching medium for the fields that oscillate within the aperture. This resistive material is intended to act as a matching load that absorbs the outgoing waves, thus considerably reducing resonance. In the following section, we test this concept with the help of the FDTD method.

\section{Loaded Apertures}

The term "loaded aperture" will be used in this work to refer to an aperture coated or surrounded by resistive sheets. The resistive sheet concept depicted in Figure 4 can be applied in an infinite number of configurations. In this work, we will confine our investigation to three configurations only. As a starting point, a resistive sheet was applied close to the shorter edges of the aperture as shown in Figure 5. This configuration will be referred to as Configuration A. Notice that to maintain the same see-through aperture area as before the application of any sheets, the actual metallic screen cutout is enlarged and the resistive sheet is effectively covering part of the aperture. As a second configuration, we placed a resistive sheet in the region surrounding the aperture such that the perimeter of the aperture will be composed of the resistive sheet, as shown in Figures 6 and 7. (Again, the actual see-through aperture area is maintained at $2 \mathrm{~mm} \times 20 \mathrm{~mm}$.) This second configuration will be referred to as Configuration $\mathrm{B}$. In the third configuration, a resistive sheet is placed on top of the conducting screen from both sides of the aperture. This placement resembles a frame-like figure as shown in Figures 8 and 9. This third configuration will be referred to as Configuration C. Notice that the area of the aperture (physical opening) remains unchanged in these configurations such that the heat transfer requirements of the shielding plane (screen) remain unaffected. Figure 10 shows a view of the aperture seen from either the interior or exterior side for Configurations A and B. The parameter $w$ in the figures designates the width of the resistive sheet.

There are infinite possibilities for the type of material used in the resistive sheets. Our choice for the material is chosen such the material either is available in the market or can be fabricated using existing technology. The material parameters for the resistive sheet will be chosen such that $\mu_{r}=1,4 \leq \varepsilon_{r} \leq 20$, and $5 \leq \sigma \leq 30 \mathrm{Ohm}^{-1} \mathrm{~m}^{-1}$.

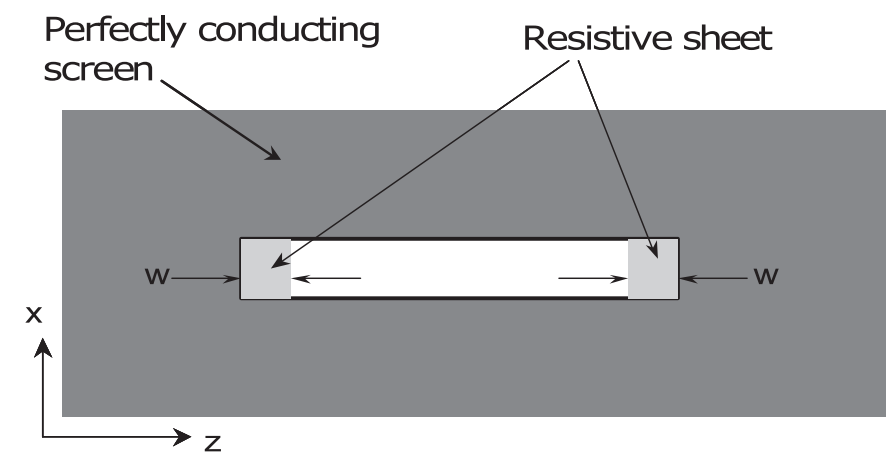

Figure 5. Configuration A. Resistive sheets are placed close to the shorter edges of the aperture. 


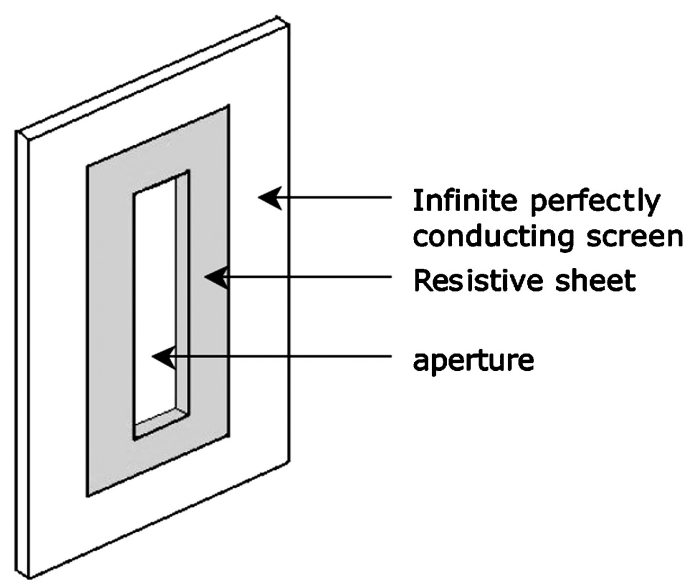

Figure 6. Configuration B. The resistive sheets constitute an inner frame that is completely contained within the aperture.

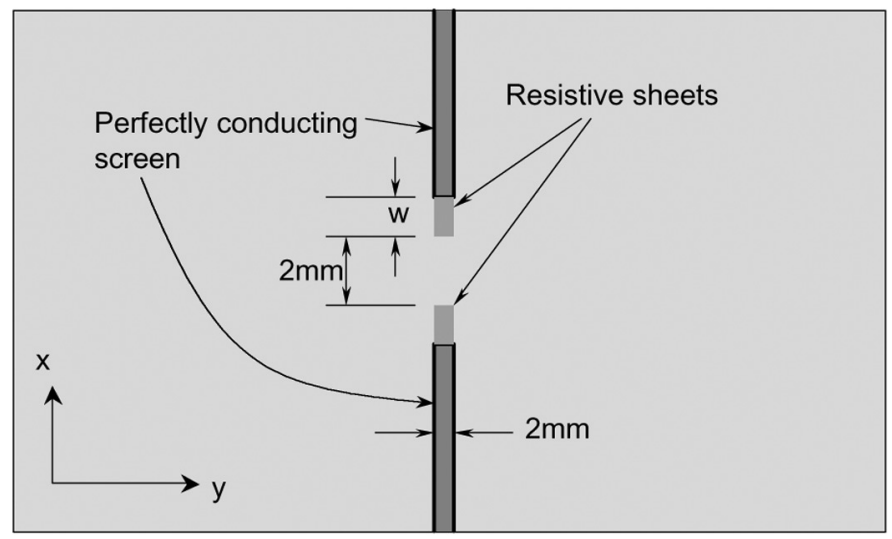

Figure 7. Cross section ( $x-y$ plane) showing the application of resistive sheets in Configuration B. Notice that the see-through aperture size and area remained unchanged.

We emphasize here that systematic optimization of the resistive sheets material property is outside the scope of this paper and is intended for future work. Furthermore, the effect of magnetic sheets is deffered to future work.

\section{FDTD Aperture Model}

The aperture considered in this study is rectangular, measuring $2 \mathrm{~mm} \times 20 \mathrm{~mm}$. The aperture is positioned in an infinite, $2 \mathrm{~mm}$ thick, perfectly conducting screen. The commercial FDTD-based code, EZ-FDTD ${ }^{\mathrm{TM}}$ is used for all the FDTD-based simulations presented in this paper. The FDTD computational model for this aperture is shown in Figure 11. The field source is an impressed current source with a differentiated Gaussian temporal profile sufficient to generate appreciable energy up to $20 \mathrm{GHz}$. The current source is polarized in the $x$-direction for maximum aperture radiation (see Figure 11). 


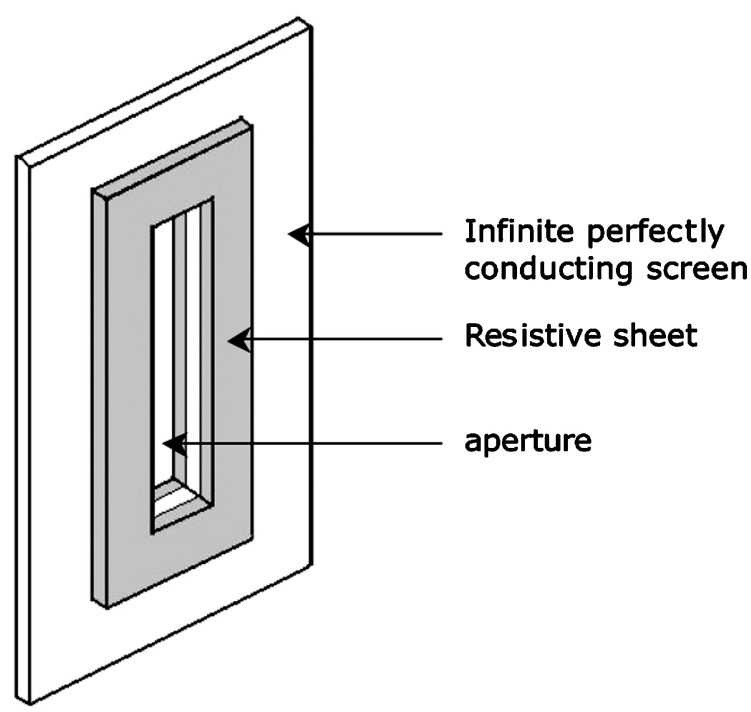

Figure 8. Configuration $C$. The resistive sheets are placed on top of the conductor from both sides.

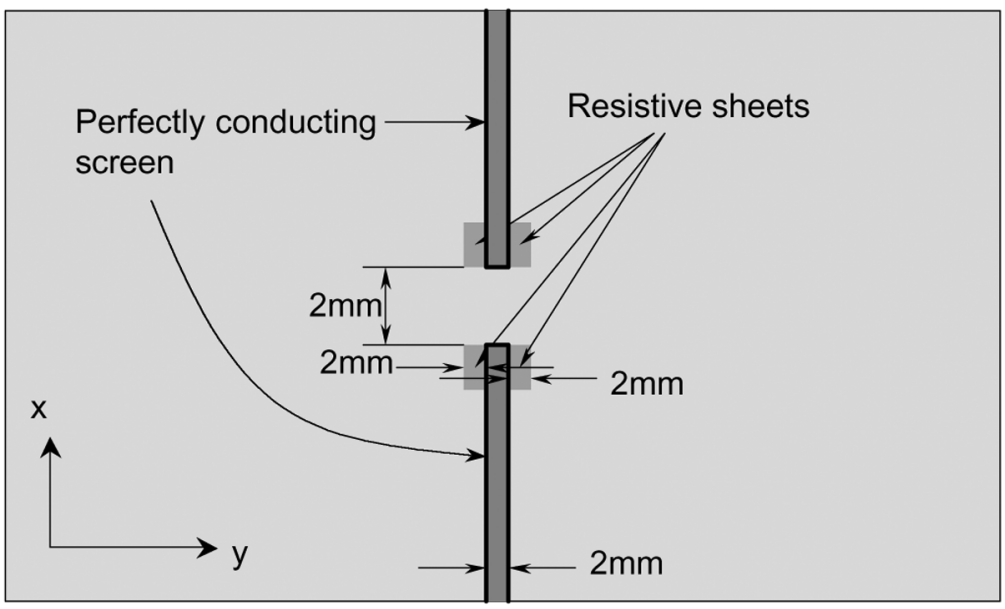

Figure 9. Cross section ( $x-y$ plane) showing the application of resistive sheets in Configuration C. Notice that the resistive sheets are applied on both sides of the screen.

The electric field is captured at the other side of the aperture and transformed to the frequency domain by Fourier transformation. The source and the monitor point are each $40 \mathrm{~mm}$ away from the plane of the aperture in opposite directions with respect to the aperture such that the source and monitor points lie along an axis that is normal to the aperture and that intersects the aperture at its center, as shown in Figure 12. The FDTD method cell size is $\Delta x=\Delta y=1 \mathrm{~mm}$ and $\Delta y$ ranges from 0.2 to $1 \mathrm{~mm}$ according to the resolution requirements of the simulation. A modified version of the perfectly matched layer (PML) is used which is capable of simulating infinite conducting screens that are positioned normally to the computational domain. (Within this implementation 


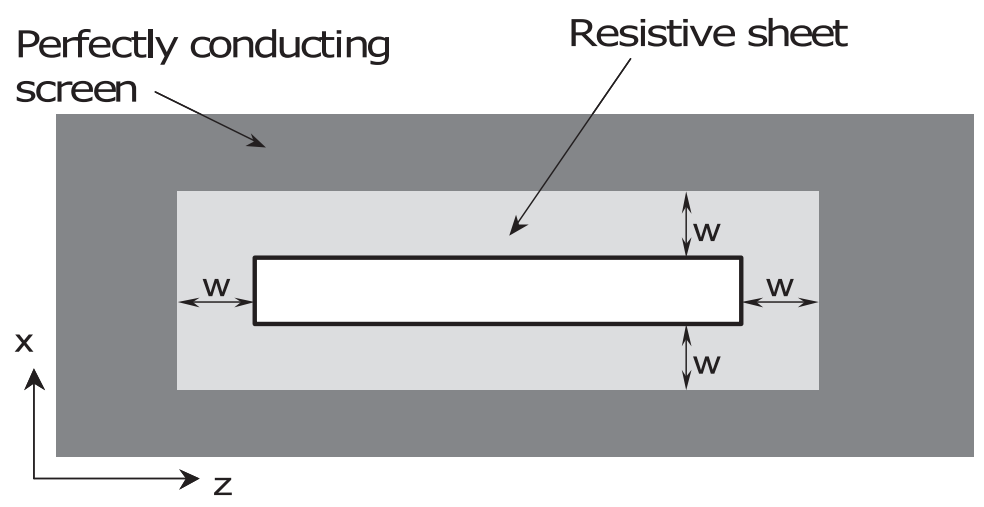

Figure 10. Front view of the aperture with resistive sheets of width $w$.

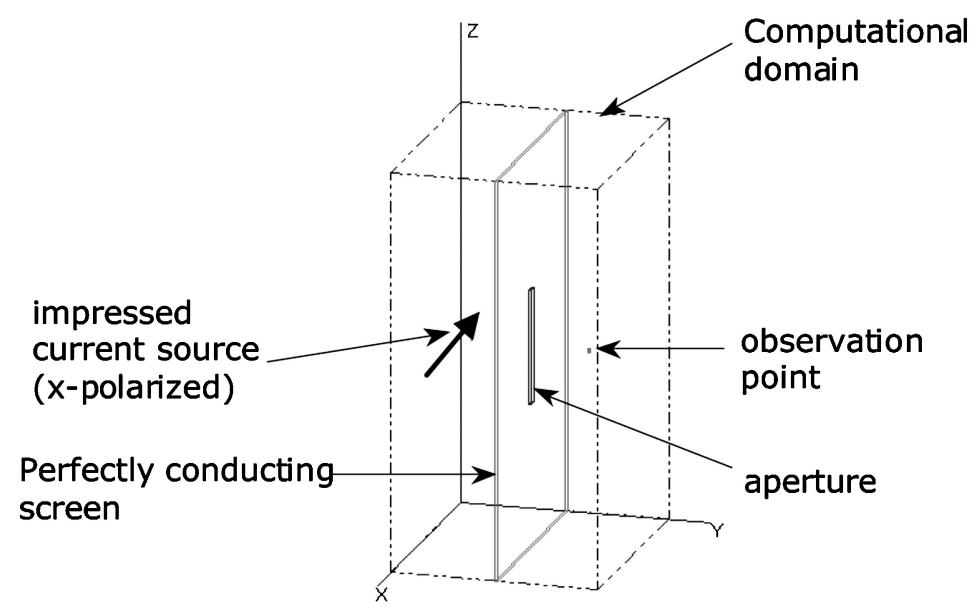

Figure 11. FDTD computational domain used for the problem of field radiation through a $2 \mathrm{~mm} \times$ $20 \mathrm{~mm}$ aperture placed in infinite perfectly conducting screen.

of the PML, in effect, any perfectly conducting sheet that is positioned normally to the computational boundary is effectively stretched to infinity.) The computational domain is positioned 50-60 $\mathrm{mm}$ away from the nearest edge of the aperture in order to minimize unwanted spurious reflections from the boundary of the computational domain.

\section{Numerical Simulation of Aperture Radiation}

When the aperture is not loaded, maximum radiation, within the frequency range of $1-12 \mathrm{GHz}$, occurs at approximately the frequency at which the aperture becomes resonant, which, for the aperture size considered here, is approximately $7.2 \mathrm{GHz}$. The resistive sheet material considered initially is given by $\mu_{r}=1, \varepsilon_{r}=4$, and $\sigma=5 \mathrm{Ohm}^{-1} \mathrm{~m}^{-1}$. In Figure 13, we present the electric field strength at the near-field monitor point $(40 \mathrm{~mm}$ away from the aperture, as depicted in Figure 12) for the $x$-polarized electric field. The same field at a distance of $160 \mathrm{~mm}$ (which we can consider as a far-field point at the aperture resonant frequency) is shown in Figure 14. The width of the resistive sheet is 


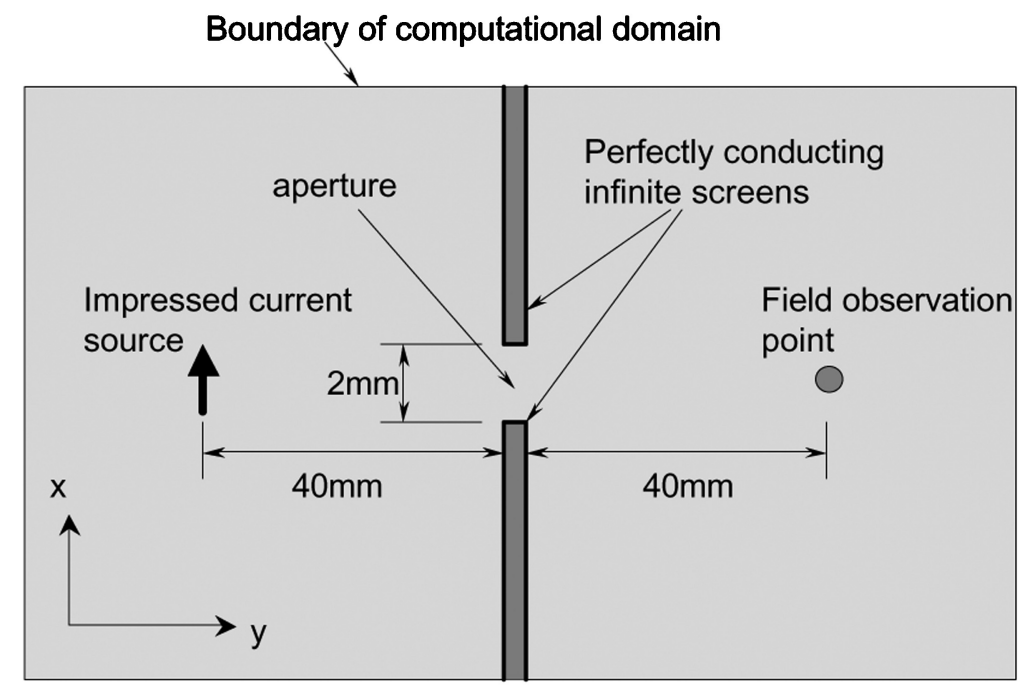

Figure 12. Cross section ( $x-y$ plane) of the FDTD computational domain showing source and field observation point placements.

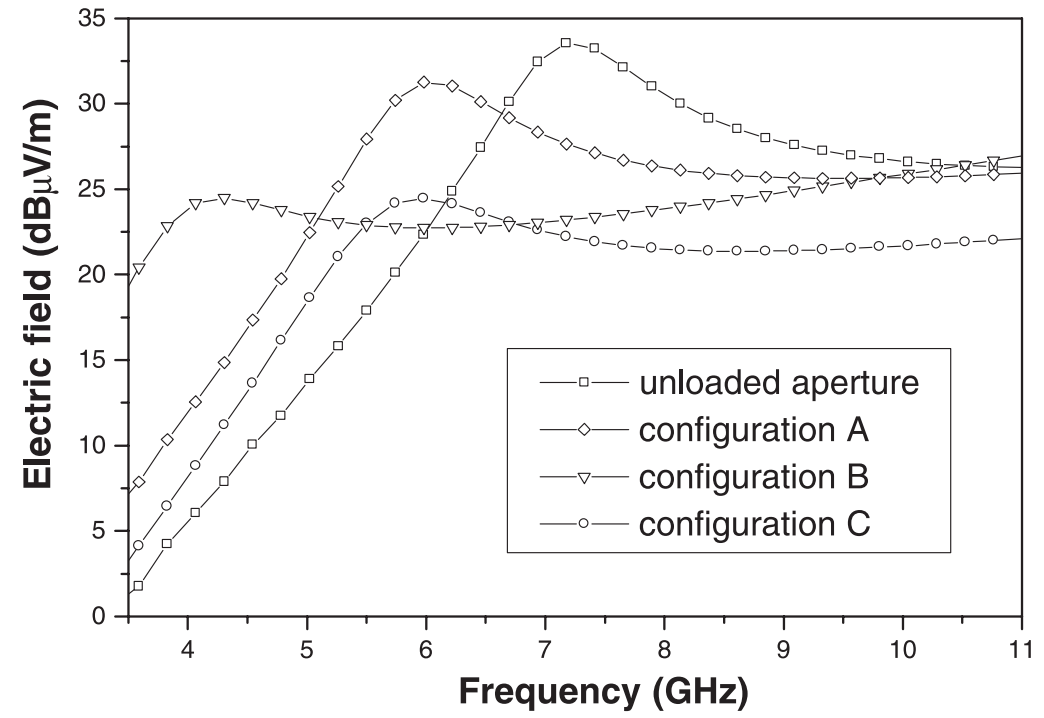

Figure 13. Electric field strength ( $x$-polarization) at a distance of $40 \mathrm{~mm}$ from the aperture.

$w=2 \mathrm{~mm}$. From Figures 13 and 14, we immediately conclude that Configuration C provides the highest field reduction in comparison to the Configurations A and B. In fact, one can observe an appreciable field reduction at the resonant frequency when Configuration $\mathrm{C}$ is used, with an approximate reduction of $12 \mathrm{~dB}$ from the case of the unloaded aperture.

Based on the transmission line model of the aperture, one would intuitively expect that Configuration A would yield the highest field reduction. It is important to keep in mind that the transmission line model (or interpretation) is only intended to provide a 


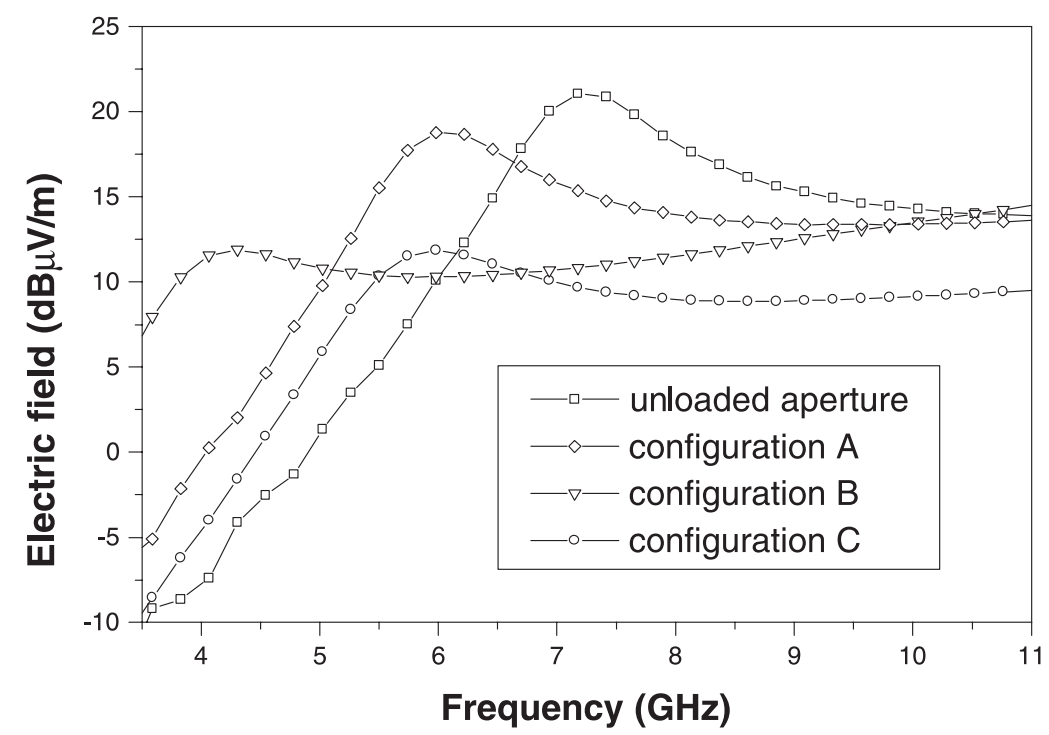

Figure 14. Electric field strength ( $x$-polarization) at a distance of $160 \mathrm{~mm}$ from the aperture.

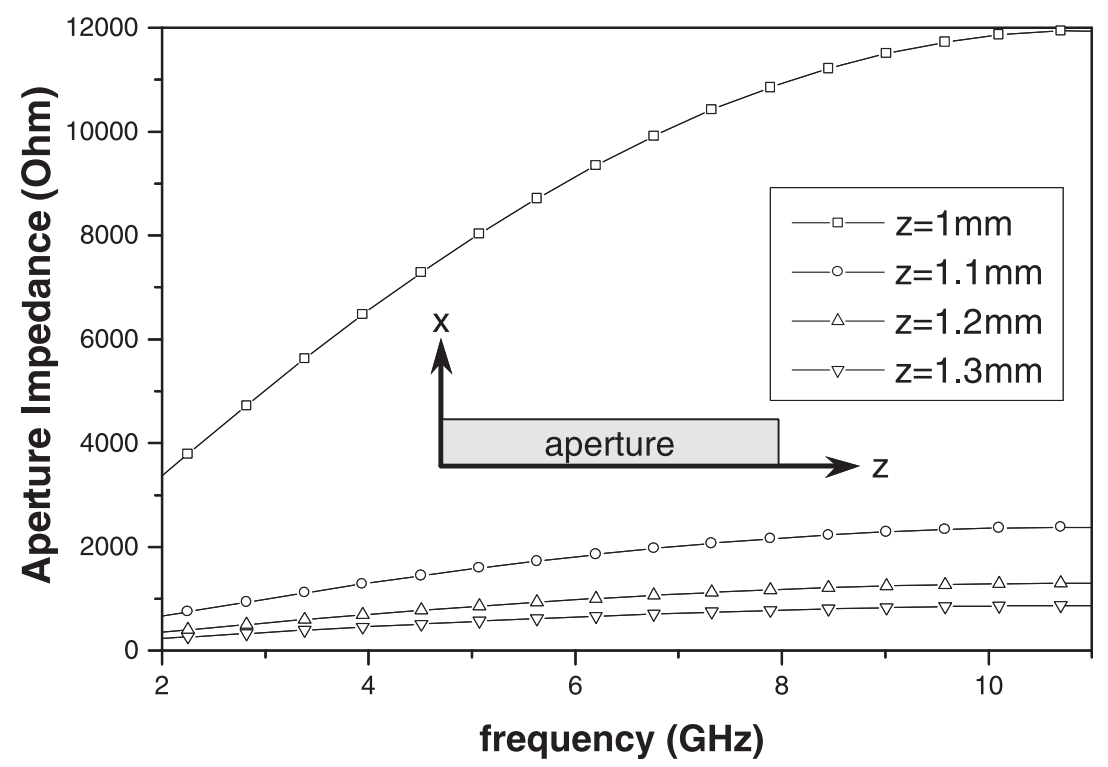

Figure 15. Aperture impedance, defined as $\left|E_{x} / H_{z}\right|$, at different locations within the aperture. $z=0$ and $z=2 \mathrm{~mm}$ correspond to the beginning and end of the aperture, respectively.

conceptual frame of reference to the field behavior within the aperture. Further insight can be gained by calculating the wave impedance within the aperture, which is defined as $\left|E_{x} / H_{z}\right|$. A simulation was conducted to test this definition of the aperture impedance, and the results, shown in Figure 15, reveal that the impedance peaks at the center of the aperture and gradually decreases as the shorter edges of the aperture are approached. The monotonically decreasing wave impedance within the aperture indicates that a tapered transmission line is a more accurate model of the aperture than a uniform line. 


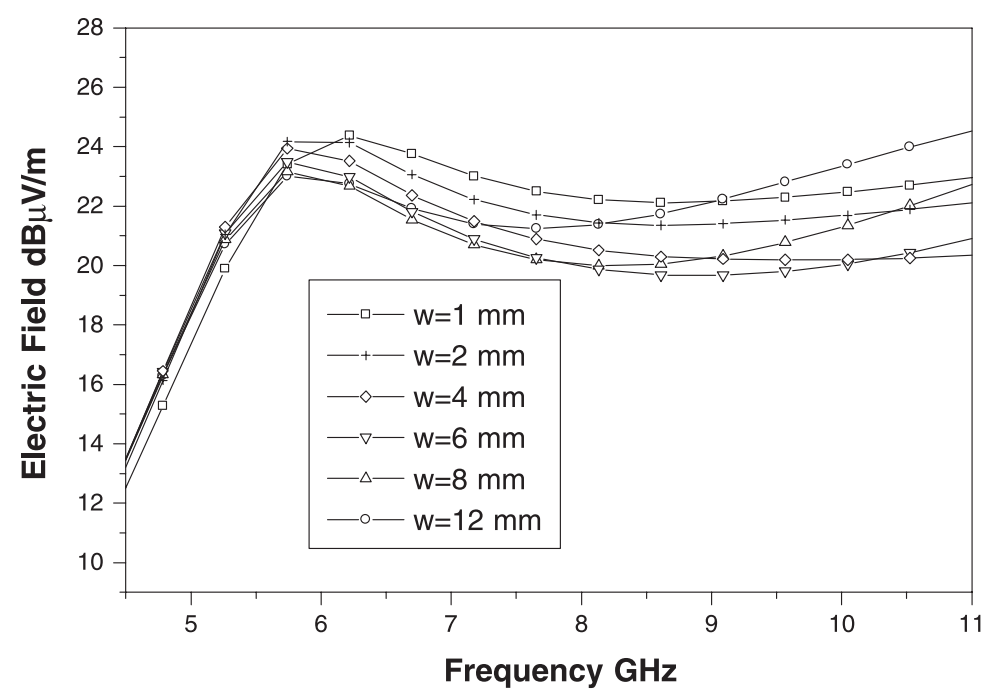

Figure 16. Effect of varying the width of the resistive sheets on the electric field strength ( $x$-polarization) for Configuration $\mathrm{C}$ with $\mu_{r}=1, \varepsilon_{r}=4$, and $\sigma=5 \mathrm{Ohm}^{-1} \mathrm{~m}^{-1}$.

The fact that Configuration $\mathrm{C}$ is more advantageous than the other configurations from the shielding effectiveness perspective is a very welcome finding. Note that it was stressed earlier that the objective of this work is to maintain a mechanically robust aperture design, which also satisfies heat transfer requirements. Notice that Configurations A and B require the presence of resistive sheets within the aperture. This requirement presents a challenge, as such configurations would require mechanical support due to the fragility of resistive sheets. Configuration $\mathrm{C}$ is mechanically stable, as the resistive sheet would be applied (coated) directly on the surface of the enclosure. For the remaining part of this paper, results will be presented only for Configuration C.

The results presented in Figures 13 and 14 were for a resistive sheet of $w=2 \mathrm{~mm}$. The effect of increasing the width from $w=1 \mathrm{~mm}$ to $12 \mathrm{~mm}$, while keeping other parameters at $\mu_{r}=1, \varepsilon_{r}=4$, and $\sigma=5 \mathrm{Ohm}^{-1} \mathrm{~m}^{-1}$, is shown in Figure 16 for the same field polarization and near-field observation point as before. Notice that the case of $w=6 \mathrm{~mm}$ offers the highest field suppression. For $w$ higher than $6 \mathrm{~mm}$, the radiated field starts to increase at and beyond the resonant frequency.

The effect of varying the conductivity, while keeping the permittivity, permeability and sheet width, $w$, constants, is shown in Figure 17. The results shown in Figure 17 for higher conductivity were obtained while insuring that the field penetration through the resistive sheet is resolved. Throughout the FDTD simulations, the cell size in the $y$-direction (normal to the resistive sheets) was maintained at or smaller than $1 / 10$ of the shortest wavelength considered in the simulation. It is noted with interest that increasing the conductivity of the resistive coating has a diminishing effect on the radiating field at or close to the resonant frequency of the aperture, while having a pronounced effect at other frequencies.

The effect of varying the permittivity while keeping the conductivity, permeability, and sheet width constant is shown in Figure 18. It is observed that varying the permittivity within the range considered has minimal effect on the radiated field.

From the above empirical analysis, we conclude that a reduction of the radiated field of up to $13 \mathrm{~dB}$ can be achieved if Configuration $\mathrm{C}$ is used with $\varepsilon_{r}$ and $\sigma$ both 


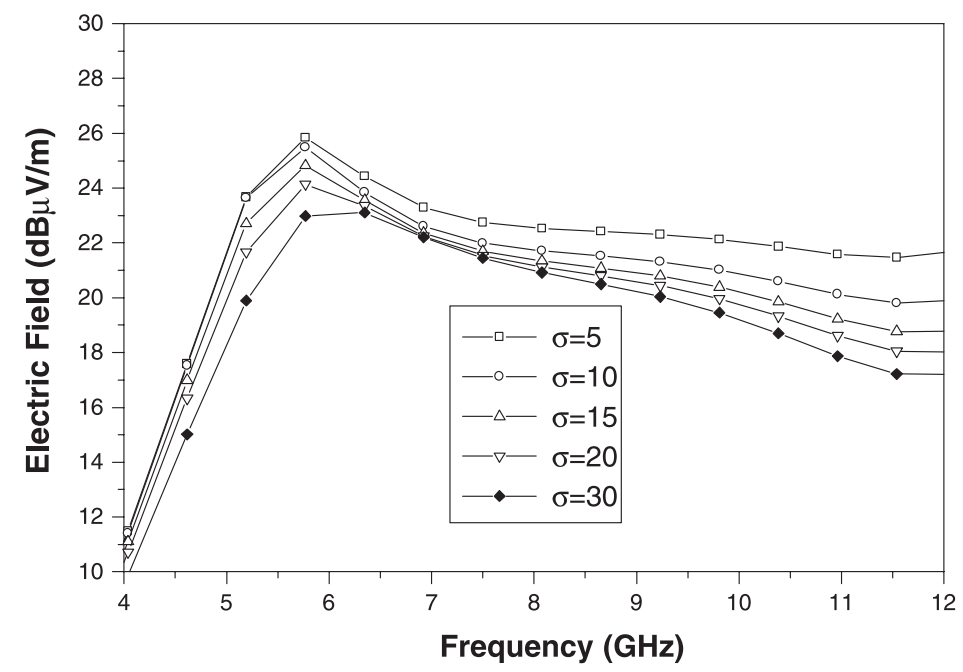

Figure 17. Effect of varying the conductivity on the electric field strength ( $x$-polarization) for Configuration $\mathrm{C}$ with $\mu_{r}=1, \varepsilon_{r}=4$, and $w=6 \mathrm{~mm}$. The conductivity, $\sigma$, is in $\mathrm{Ohm}^{-1} \mathrm{~m}^{-1}$.

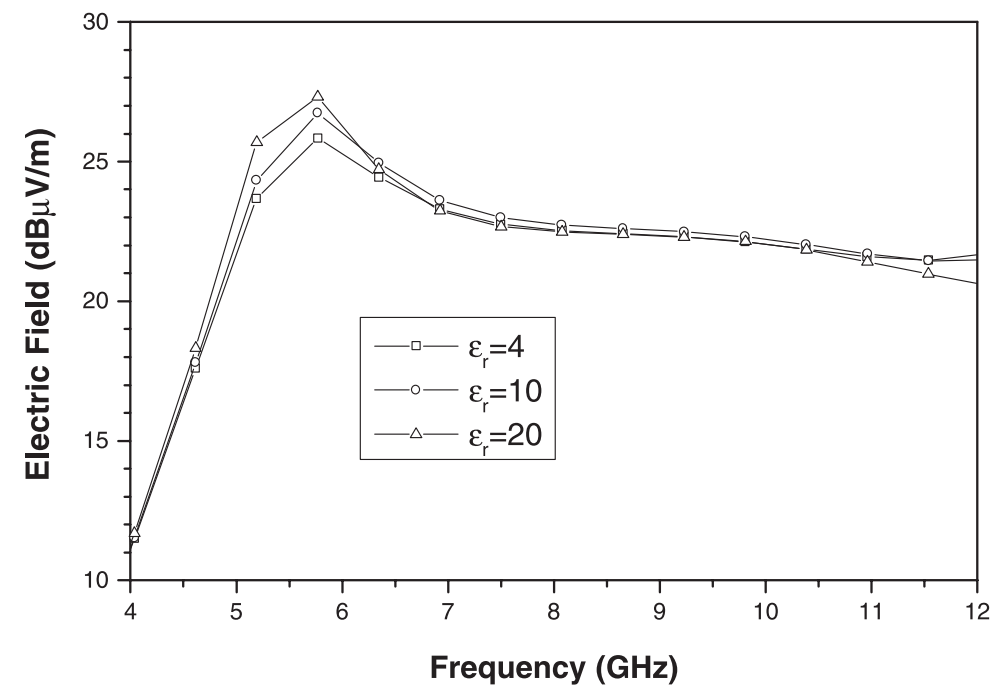

Figure 18. Effect of varying the permittivity on the electric field strength ( $x$-polarization) for Configuration $\mathrm{C}$ with $\mu_{r}=1, \sigma=5 \mathrm{Ohm}^{-1} \mathrm{~m}^{-1}$, and $w=6 \mathrm{~mm}$.

lying within the ranges considered, and with a sheet width, $w$, of $6 \mathrm{~mm}$. Among the material design parameters considered in this study, the width of the sheet was found to be the most sensitive factor in affecting the shielding effectiveness of the coated aperture. Based on the results presented in Figure 16, the relationship between the loaded aperture radiation and sheet width is a complex one and is clearly highly frequency dependent. It is important to keep in mind that the radiation through the aperture is due to the direct field penetration through the aperture and is also due to the electric current that is induced on the external side of the screen, especially in the immediate vicinity of the aperture. In Figures 19a and 19b, we show a calculation of the electric current density 


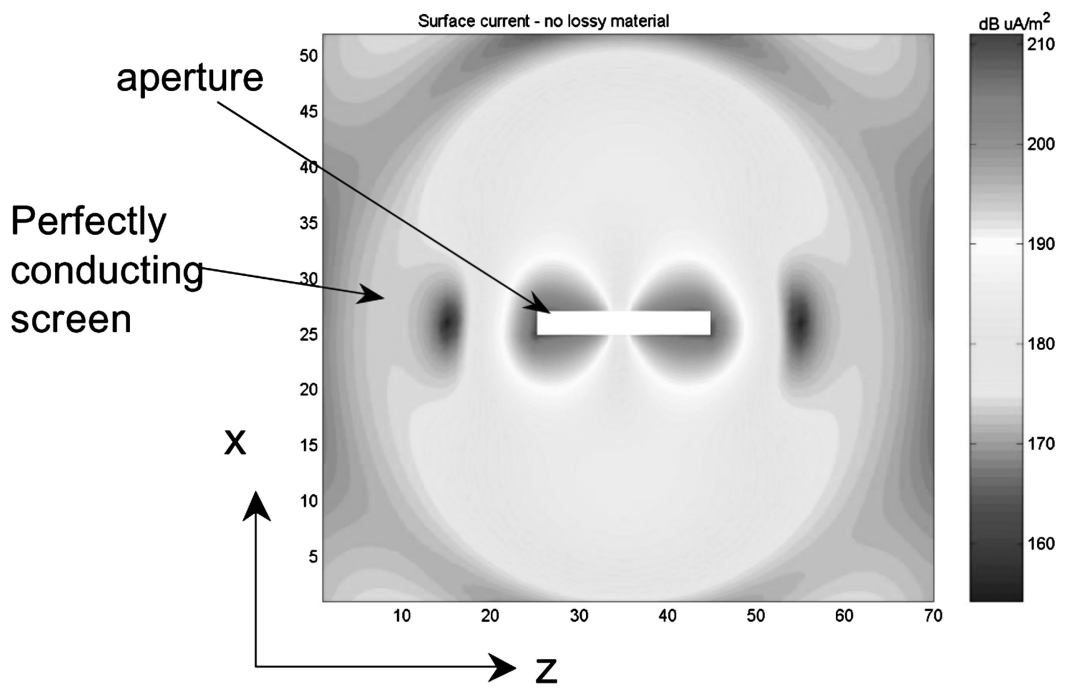

(a)

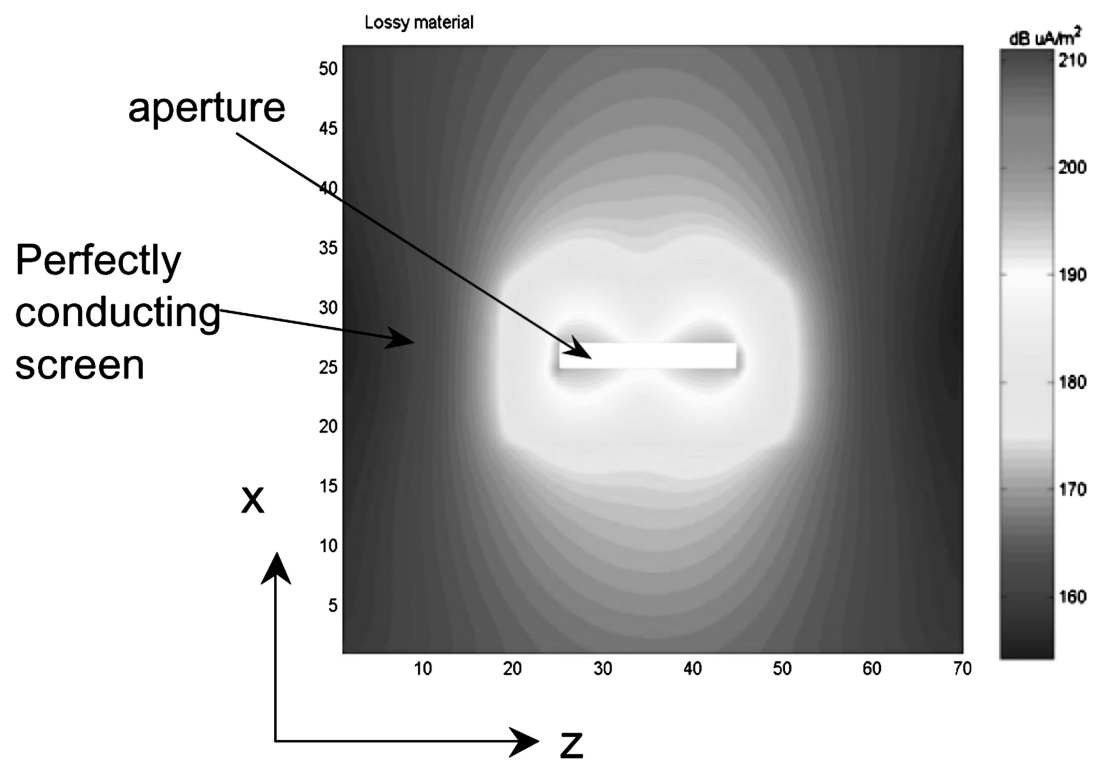

(b)

Figure 19. Total surface current density at the external surface of the screen at $7.2 \mathrm{GHz}$. (a) Unloaded aperture; (b) loaded aperture: Configuration $\mathrm{C}$ with $\mu_{r}=1, \varepsilon_{r}=4, \sigma=5$, and $w=6 \mathrm{~mm}$. 
for two cases of unloaded and loaded apertures. In Figure 19b, we show the current density for Configuration $\mathrm{C}$, where the resistive sheet properties are $\mu_{r}=1, \varepsilon_{r}=4$, $\sigma=5 \mathrm{Ohm}^{-1} \mathrm{~m}^{-1}$, and $w=6 \mathrm{~mm}$. Notice that the dipole-like behavior of the unloaded aperture is clearly visible in Figure 19a. The effect of loading the aperture is shown to dramatically reduce the electric current density on the external side of the screen, thus leading to reduced external radiated field.

\section{Summary and Discussion}

This paper presents the novel use of resistive sheets to reduce electromagnetic penetration through apertures while maintaining robust mechanical design. The use of resistive sheets or coating was motivated by the interpretation of the aperture and the oscillating field within the aperture as a transmission line system. Based on this interpretation, a resistive material was applied in different configurations to minimize aperture resonance. Several resistive sheet configurations were tested numerically using the FDTD method. One of the configurations tested was found to yield an aperture field strength reduction of up to $13 \mathrm{~dB}$ in comparison to the case of the unloaded aperture. Such appreciable reduction in the field was made possible using only a $6 \mathrm{~mm}$ wide strip of a resistive sheet on both sides of the aperture. Finally, it is emphasized that this study is empirical. A more exhaustive optimization is the subject of a future study in which the material properties and topology of the resistive sheets are considered.

As a final note, we make a comparison between the loaded aperture discussed in this work and the work performed previously on loaded monopole and dipole antennas. In earlier works, resistive loading, consisting of coating an antenna with lossy material, was used with great effectiveness in increasing the broadband potential of the antenna and thus its potential for effective matching to a purely resistive transmission line system (there are numerous publications on the use of resistive coating of antennas; the interested reader is advised to read the original paper on this subject by Wu and King, 1965). Based on Babinet's principle, the aperture is the dual of the monopole, and one would expect that a resistively loaded aperture, in the context of the definition used in this work, would have an analogous effect to that of the resistively loaded dipole. In fact, loading the dipole typically achieves a broadband behavior, however, at the cost of decreased efficiency. It is interesting that one notices a highly similar scenario in the case of the loaded aperture. Careful observation of Figure 13, for instance, shows an increase in the broadband potential for the loaded aperture, i.e., an increased radiation at frequencies below resonance while an appreciable decrease in radiation at resonance. It is obvious that our objective in this work was to reduce radiation at resonance, but one can also think of this objective as widening the impedance bandwidth of the aperture.

\section{References}

Archambeault, B. 1997. Modeling of the electromagnetic radiation from shielded enclosures with apertures and attached wires in a real-world environment. Ph.D. Dissertation, University of New Hampshire.

Belokour, I., J. LoVetri, \& S. Kashyap. 2001. A higher-order mode transmission line model of the shielding effectiveness of enclosures with apertures. EMC International Symposium on Electromagnetic Compatibility 2:702-707.

Bethe, H. A. 1944. Theory of diffraction by small holes. Phys. Rev. 66:163-182.

Butler, C. M., Y. Rahmat-Samii, \& R. Mittra. 1978. Electromagnetic penetration through apertures in conducting surface. IEEE Trans. Antennas Propagat. 26(1):82-93. 
Cerri, G., R. De Leo, \& V. M. Primiani. 1992. Theoretical and experimental evaluation of the electromagnetic radiation from apertures in shielded enclosures. IEEE Trans. Electromagnetic Compatibility 34(4):423-432.

Crew, H. 1900. Scientific memoirs: The wave theory of light. Chicago: American Book Corp.

Fresnel, A. J. 1819/1900. Mémoire sur la diffraction de la lumière. Annales de Chimie et de Physique, t.x., 288. Also in Scientific Memoirs, 1900, 79-144, ed. H. Crew. Chicago: American Book Corp.

Gardner, C. L., \& G. I. Costache. 1995. The penetration of EM waves through loaded apertures. IEEE Trans. Electromagnetic Compatibility 37(3):358-366.

Georgakopoulos, S. V., C. R. Birtcher, \& C. A. Balanis. 2001. Coupling modeling and reduction techniques of cavity-backed slot antennas: FDTD versus measurements. IEEE Trans. Electromagnetic Compatibility 43(3):261-272.

Harrington, R. F., \& J. R. Mautz. 1976. A generalized network formulation for aperture problems. IEEE Trans. Antennas Propagat. 24(6):870-873.

Hill, D. A., M. T. Ma, A. R. Ondrejka, B. F. Riddle, \& M. L. Crawford. 1994. Aperture excitation of electrically large, lossy cavities. IEEE Trans. Electromagnetic Compatibility 36(3):169-178.

Huygens C. 1690/1900. Traité de la luminère. Leyden. In Scientific memoirs, 1900, 3-41, ed. H. Crew. Chicago: American Book Corp.

Li, M., J. Nuebel, J. L. Drewniak, R. E. DuBroff, T. H. Hubing, \& T. P. VanDoren. 2000. EMI from cavity modes of shielding enclosures-FDTD modeling and measurements. IEEE Trans. Electromagnetic Compatibility 42(1):29-38.

Li, M., J. Nuebel, J. L. Drewniak, T. H. Hubing, R. E. DuBroff, \& T. P. Van Doren. 2000a. EMI from airflow aperture arrays in shielding enclosures-experiments, FDTD, and MoM modeling. IEEE Trans. Electromagnetic Compatibility 42(3):265-275.

Li, M., J. Nuebel, J. L. Drewniak, T. H. Hubing, R. E. DuBroff, \& T. P. Van Doren. 2000b. EMI reduction from airflow aperture arrays using dual-perforated screens and loss. IEEE Trans. Electromagnetic Compatibility 42(42):135-141.

Olyslager, F., E. Laermans, D. De Zutter, S. Criel, R. De Smedt, N. Lietaert, \& A. De Clercq. 1999. Numerical and experimental study of the shielding effectiveness of a metallic enclosure. IEEE Trans. Electromagnetic Compatibility 41(3):202-213.

Ott, H. W. 1998. Noise reduction techniques in electronic systems, 2nd ed. New York: Wiley.

Robinson, M. P., T. M. Benson, C. Christopoulos, J. F. Dawson, M. D. Ganley, A. C. Marvin, S. J. Porter, \& D. W. P. Thomas. 1998. Analytical formulation for the shielding effectiveness of enclosures with apertures. IEEE Trans. Electromagnetic Compatibility 40(3):240-248.

Tanabe, S., N. Nagano, T. Itoh, Y. Murata, \& S. Mizukawa. 1996. 3D-FEM analysis for shielding effects of a metallic enclosure with apertures. IEEE International Symposium on Electromagnetic Compatibility 375-380.

U.S. Department of Defense Multi-University Research Initiative Program on Effects of Radiofrequency Pulses on Electronic Circuits and Systems. 2001. http://www.ireap.umd.edu/ MURI-2001/

Wu, T. T., \& R. W. P. King. 1965. The cylindrical antenna with nonreflecting resistive loading. IEEE Trans. Antennas Propagat. 13(1). 